\title{
Salvage coronary artery bypass after failed stent insertion in adult Kawasaki disease
}

\author{
Lalit Kapoor, ParthoProtim Chowdhury, Dhiraj Barman, Rakesh Gayen, Vikash Toshniwal, Satish Kumar, \\ Pradeep Narayan
}

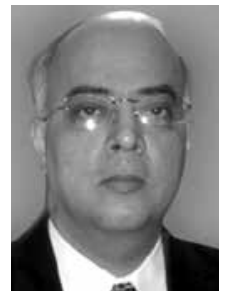

NH Rabindranath Tagore International Institute of Cardiac Sciences, Kolkata, India

Kardiochir Torakochir Pol 2020; 17 (3): 168-169

Kawasaki disease is more common in children but can occasionally present in late adulthood as obstructive coronary artery disease, which is a chronic sequela of the condition. Revascularization is often required in these patients. The decision making can be difficult because of presence of concomitant aneurysms. Attempted percutaneous coronary intervention $(\mathrm{PCl})$ of coronary artery lesions in Kawasaki disease in adults can be challenging and fraught with complications and coronary artery bypass grafting (CABG) should be preferred.

We report a case of a 44-year-old patient presenting with obstructive coronary artery lesions where an attempted $\mathrm{PCl}$ was associated with complications and a salvage CABG needed to be carried out.

A 44-year-old man presented with acute ST elevation myocardial infarction. He was non-diabetic, non-hypertensive and a nonsmoker but had a clinical diagnosis of Kawa-

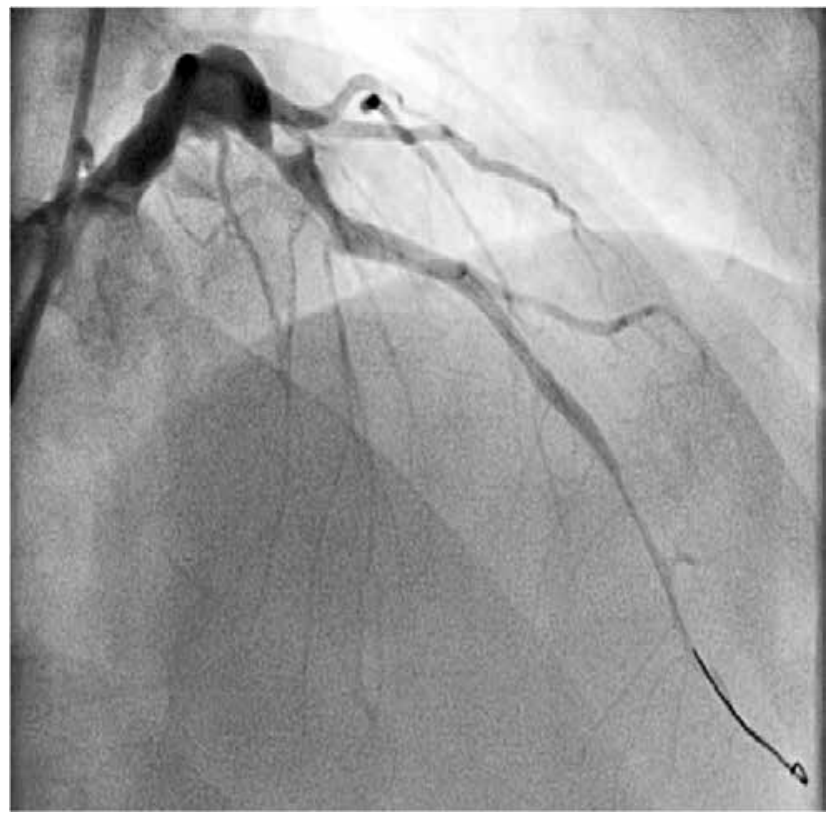

Figure 1. Angiogram showing stenosis in LAD saki disease made in his childhood. Coronary angiography showed an aneurysm involving the left main stem (LMS) and ostio-proximal left anterior descending artery (LAD), followed by a tight lesion of the LAD. The right coronary artery territory was normal (Figures 1 and 2).

$\mathrm{PCl}$ was attempted. The LAD was dilated but during insertion the stent $(3.5 \times 10 \mathrm{~mm})$ got dislodged and was partially deployed in the aneurysmal part of the distal LMS and the proximal LAD. Only a TIMI 3 flow was achieved at this stage in the LAD and the patient was referred for urgent surgical intervention. Because of the partially deployed stent in the aneurysmal LMS it was decided to graft both the LAD and circumflex territories. As the patient was hemodynamically stable, a left internal thoracic artery (LITA)-right internal thoracic artery (RITA) composite graft created in a $Y$ fashion was used. The LITA was anastomosed to the LAD and the RITA to the ramus inter-

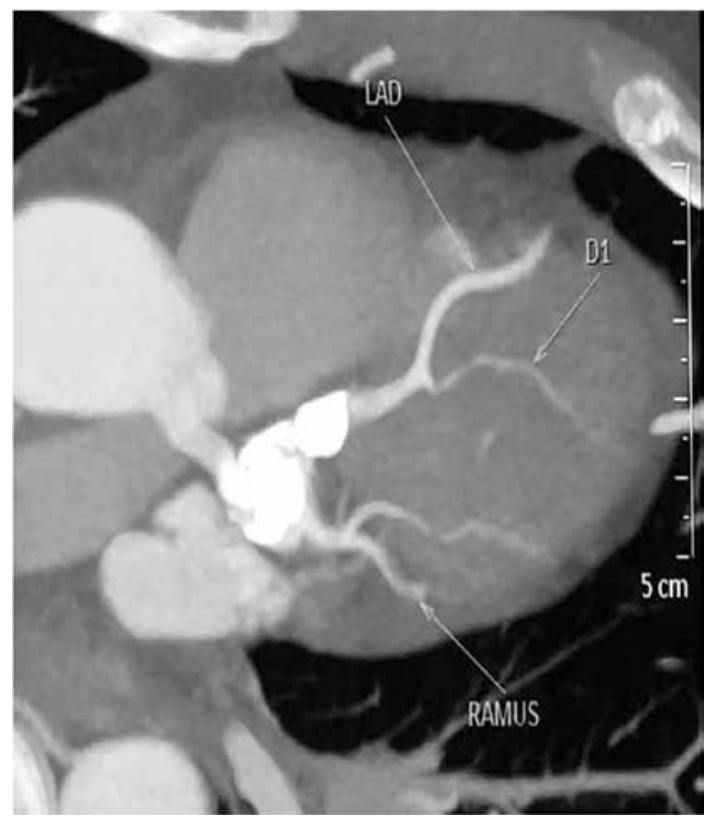

Figure 2. Computed tomography scan showing aneurysmal left main stem stenosis

Address for correspondence: Pradeep Narayan, NH Rabindranath Tagore International Institute of Cardiac Sciences, Kolkata, India, phone: +91 8017249115, e-mail: pradeepdoc@gmail.com

Received: 11.05.2020, accepted: 9.07.2020. 
medius and obtuse marginal, sequentially on the beating heart.

The second issue was to address the aneurysmal segment of the LMS with a stent in situ which would be prone to clot formation and distal embolization. The distal end of the aneurysm was snugged and as the heart was beating, it was possible to assess the impact of attempted ligation on the ECG before tying the artery completely. The proximal end of the aneurysm at the LMS-LAD junction could not be ligated as there was an under-deployed stent in situ extending into the LAD.

Thus concerns remained over the potential thrombogenicity and proximal embolization of clot into the aorta and resultant neurological morbidity.

Aortic cross clamping with a short period of fibrillatory arrest was undertaken with venting of the LV through the right superior pulmonary vein (RSPV) in preference to antegrade and retrograde cardioplegia. An aortotomy was performed and the left main ostium closed using a pericardial patch sutured with 5-0 polypropylene. The patient made an uneventful recovery and remains well.

The importance of Kawasaki disease producing coronary artery disease in adults remains underestimated. In fact, after atherosclerosis Kawasaki disease is the second most common cause of coronary artery disease in adults [1] and is responsible for myocardial infarction in $5 \%$ of adults under the age of 40 [2]. Median age of patients presenting for CABG in adulthood secondary to Kawasaki disease is 28 [3].

While destruction of the internal elastic lamina, necrosis of smooth muscle cells, and myointimal proliferation are responsible for developing aneurysms or ectasia [4], stenotic lesions result due to luminal myofibroblastic proliferation and thrombosis [1].

In the presence of ischemic symptoms, inducible myocardial ischemia or $>75 \%$ stenosis of proximal coronary arteries revascularization with $\mathrm{PCl}$ or CABG is recommended. Covered stents limit further expansion of involved coronary segments; however, because of dense fibrosis and calcification in the arterial wall relatively high balloon pressures are often necessary. As a result, it has been suggested that if the balloon pressure of $10 \mathrm{~atm}$ or more is required then either rotational ablation or bypass surgery should be performed instead [5]. CABG is also recommended for larger lesions and for associated obstructive coronary artery disease, multiple coronary lesions and severe LV dysfunction $[1,5]$. Catheter intervention is specifically contraindicated for individuals who have vessels with multiple, ostial, or long-segment lesions [5]. However, aneurysmal segments make dislodgement of stents more likely and careful evaluation of the case should be undertaken to assess the suitability for $\mathrm{PCl}$. In our case, because of recent ST-elevation myocardial infarction (STEMI) PCI was attempted, with an unsatisfactory outcome.

CABG in adult patients with Kawasaki disease is similar to atherosclerotic disease with respect to conduit selection. However, an important difference lies in administration of cardioplegia and dealing with the aneurysmal segment. The aneurysmal segment is prone to thrombus formation and risk of distal embolization during cardioplegia should be borne in mind. The risk was higher in our case where there was a partially deployed stent in situ.

CABG is often required in children but presentation of obstructive coronary artery disease in adulthood is relatively less common and presentation in the $5^{\text {th }}$ decade is extremely rare.

$\mathrm{PCl}$ in Kawasaki disease has limited indications and in the sub-set of coronary artery disease secondary to Kawasaki disease it is important to have a heart team approach to decide the most suitable approach to revascularization. Off pump coronary artery bypass (OPCAB) where possible may be preferable in presence of clots in the aneurysmal segment.

\section{Disclosure}

The authors report no conflict of interest.

\section{References}

1. ElGuindy MS, ElGuindy AM. Aneurysmal coronary artery disease: an overview. Glob Cardiol Sci Pract 2017; 2017: e201726.

2. Daniels LB, Tjajadi MS, Walford HH, et al. Prevalence of Kawasaki disease in young adults with suspected myocardial ischemia. Circulation 2012; 125: 2447-2453.

3. Tsuda E, Abe T, Tamaki W. Acute coronary syndrome in adult patients with coronary artery lesions caused by Kawasaki disease: review of case reports. Cardiol Young 2011; 21: 74-82.

4. Burgner D, Davila S, Breunis WB, et al. International Kawasaki Disease Genetics Consortium. A genome-wide association study identifies novel and functionally related susceptibility Loci for Kawasaki disease. PLoS Genet 2009; 5: e1000319.

5. Newburger JW, Takahashi M, Gerber MA, et al. Committee on Rheumatic Fever, Endocarditis and Kawasaki Disease; Council on Cardiovascular Disease in the Young; American Heart Association; American Academy of Pediatrics. Diagnosis, treatment, and long-term management of Kawasaki disease: a statement for health professionals from the Committee on Rheumatic Fever, Endocarditis and Kawasaki Disease, Council on Cardiovascular Disease in the Young, American Heart Association. Circulation 2004; 110: 2747-2771. 